\title{
Sleep Telemedicine: Patient Satisfaction and Treatment Adherence
}

\author{
Roshni Parikh, M.D., ${ }^{1}$ Megan Nicole TouVelle, ${ }^{2}$ \\ Huaping Wang, Ph.D., ${ }^{1}$ and Sarah Nath Zallek, M.D. ${ }^{1-3}$ \\ ${ }^{1}$ University of Illinois College of Medicine, Peoria, Illinois. \\ ${ }^{2}$ OSF St. Francis Medical Center, Peoria, Illinois. \\ ${ }^{3}$ INI Sleep Center, Illinois Neurological Institute, Peoria, Illinois.
}

\begin{abstract}
Objective: Obstructive sleep apnea is common, but access to diagnosis remains limited. Telemedicine may allow greater access to care; however, its effect on patient satisfaction and treatment adherence is unknown. This study compares patient satisfaction and continuous positive airway pressure (CPAP) adherence of patients seen by videoconference with those seen in person. Materials and Methods: New patients seen via video or in person at a sleep center completed a survey, with three questions pertaining to satisfaction with the provider. Questions were scored 1-5; the sum was the patient satisfaction score. CPAP adherence was retrospectively analyzed in patients who met the physician via video or in person. Percentage of nights CPAP was used for $\geq 4 h$ and average minutes of CPAP use per night over 2 consecutive weeks were compared. Results: A Mann-Whitney test compared patient satisfaction of the 90 subjects (of whom, 56 met physician in person and 34 via video). Mean scores (in person, 14.82; video, 14.91; $\mathrm{p}=0.851$ ) did not differ between groups. Mann-Whitney tests compared CPAP adherence in the 172 subjects (of whom, 111 met physician in person and 61 via video). Mean percentage of nights CPAP was used $\geq 4 h$ (in person, $71 \%$; video, 65\%; $\mathrm{p}=0.198$ ) and the average minutes per night of CPAP use (in person, 340.55; video, 305.31; $\mathrm{p}=0.153$ ) did not differ between groups. Conclusions: The findings indicate that patients were equally satisfied with their provider and adherent to CPAP treatment whether they were seen in person or via video. Videoconferencing may improve access to patient care without reducing patient satisfaction or treatment adherence.
\end{abstract}

Key words: telemedicine, telehealth, e-health

\section{Introduction}

$\mathrm{T}$ he use of technology is increasing in nearly every field, and medicine is no exception. Telemedicine as a means of remote patient-physician interaction is growing. ${ }^{1}$ This technology may improve patient access to care. ${ }^{2}$ Telemedicine is being used in a variety of medical arenas, including psychiatric counseling, ${ }^{3,4}$ home healthcare services, ${ }^{5,6}$ intensive care units (ICUs), ${ }^{7}$ and emergency stroke care. ${ }^{8}$
Access to specialists who are limited in number may be a particularly valuable benefit of telemedicine. Joint teleconsultation with the general practitioner, specialist, and patient have recently become popular. Virtual consultations with specialists can eliminate "duplication of investigations and treatments." 0 verall cost for the patient or the provider may be greater or lower with the use of teleconsultations; however, all potential benefits of telemedicine should be considered, including patient satisfaction, efficiency, and timeliness of care. Joint teleconsultations with a general practitioner and specialist have produced higher patient satisfaction and increased the likelihood of follow-up care. ${ }^{9,10}$

Telemedicine is also being used in follow-up care. Morgan et al. provided parents of children diagnosed with severe congenital heart disease with either home videoconferencing capabilities or telephone follow-up calls as a means to communicate with their physician postdischarge. All families provided with videoconferencing found it beneficial. ${ }^{2}$ Salvador et al. showed that general practitioners can access home coagulometer INR results via electronic record to monitor Warfarin dose without requiring frequent visits to healthcare centers. $^{5}$

Rural communities may lack the most advanced technology or the specialists necessary in emergency situations. For example, rural hospitals in northern Ontario use videoconferencing between their referring physician and a neurologist at a specialized care center to aid in assessing patients' neurological symptoms in the setting of an acute stroke. ${ }^{8}$ Telemedicine is also useful in ICU settings by enabling an intensivist, a critical care nurse, and a clerical person to round on patients and make critical decisions via an eICU equipped with bedside monitors and microphones. ${ }^{7}$

\section{APPLICATIONS OF TELEHEALTH IN SLEEP MEDICINE}

Obstructive sleep apnea (OSA) is "a condition characterized by repetitive obstructions of the upper airway often resulting in oxygen desaturation and arousals from sleep." 11 Typical symptoms of OSA include snoring, restless sleep, and daytime sleepiness or fatigue. ${ }^{12}$ Approximately $9 \%$ of women and $24 \%$ of men have undiagnosed moderately severe sleep-disordered breathing. ${ }^{13}$ OSA has been associated with numerous cardiovascular complications including coronary heart disease, heart failure, hypertension, and stroke. ${ }^{14}$ Predisposing factors for OSA include obesity, male gender, nasal obstruction, endocrine abnormalities, craniofacial abnormalities, and familial history.

OSA can be diagnosed using nocturnal polysomnography (PSG) in a sleep laboratory, home PSG, or more limited portable monitors. ${ }^{15,16}$ Full PSGs are very expensive, whereas portable monitors can be more economical. The American Academy of Sleep Medicine recommends 


\section{PARIKH ET AL.}

that portable monitors may be used in a lab or in a patient's home under strict guidelines, but that an in-laboratory PSG is the preferred option for most patients. ${ }^{16}$

There are four main treatments for OSA: continuous positive airway pressure (CPAP), weight loss, oral appliances, and surgery. ${ }^{17-19}$ CPAP is the most widely accepted form of treatment; however, maximizing adherence is one of the most important challenges sleep specialists face. ${ }^{17,20}$

OSA is under diagnosed. ${ }^{13}$ The relatively limited supply of sleep specialists is likely to be a continued barrier to diagnosis. Telemedicine may be a very efficient way to allow greater access to care.

Telemedicine has been extensively used in the field of sleep medicine. Specifically, telemedicine can be used to transfer polysomnograms online from remote sites to larger facilities equipped with specialists capable of study interpretation. Kristo et al. reported that this is not only feasible but can even be cost-effective. ${ }^{21}$ Athome sleep studies can be effectively conducted via home monitoring systems, eliminating the need for overnight sleep studies at a facility. Such studies can be conducted in real time or with portable monitoring that is later downloaded for interpretation. Sleep studies in real time can use video technology to connect the patient to a sleep technologist. $^{22}$

Additionally, telemedicine can be used to improve CPAP adherence. Smith et al. conducted a study in which telehealth units allowed nurses to view and educate patients in their homes. This study showed how a structured system of telecommunication with patients improved CPAP compliance. ${ }^{6}$

To have sustainable telemedicine programs, there must be acceptance and understanding among patients and caregivers. Users must believe that telemedicine is useful and adds value to their medical care. ${ }^{23}$ The study by Swinton et al. found that primary care physicians and patients alike expressed belief that telehealth is "an acceptable solution to the access to care problem in the treatment of depression in rural communities." 24 Timmerberg et al. conducted a pilot study in which dieticians counseled diabetic patients via videoconference. Results showed patients were equally satisfied with counseling via videoconference and in person. Patients said they were comfortable talking via videoconference, found the lack of physical contact acceptable, generally found the encounter convenient, and had a satisfaction rating of 4.6 out of $5 .{ }^{25}$ A second study using videoconferencing showed that $96 \%$ of psychiatric patients were comfortable communicating with an off-site clinician. In addition, a majority of the patients stated they would participate in videoconferencing again. ${ }^{26}$

Although telemedicine has been explored in various ways, few studies have been conducted that use direct physician-patient videoconferencing in sleep medicine. This study was designed to compare the effectiveness of videoconferencing versus in-person interactions in sleep medicine with respect to patient satisfaction and sleep apnea treatment adherence. Patient satisfaction and adherence among sleep patients may differ from other fields, possibly because sleep visits are fairly history intensive and less dependent on physical examination, although examination is an essential component of the visit. If these two factors are similar between the two groups, sleep specialist physicians may be encouraged to apply telemedicine to increase patient access to specialty care.

\section{Materials and Methods}

The Illinois Neurological Institute (INI) Sleep Center has two locations, one in Peoria, IL, and one in Morton, IL. At both locations the patient is first seen by a nurse practitioner (NP) to obtain initial history, conduct a physical examination, and enter this information and an initial impression and plan in the electronic medical record (EMR). The patient is then seen by a sleep physician, either in person or by videoconference. If the patient is at the Morton center and the sleep physician is at the Peoria center at that time, the NP calls the sleep physician in Peoria by telephone to present and discuss the patient. During that conversation, the sleep physician reviews the information entered into the EMR and makes changes based on the discussion with the NP if appropriate. The Polycom communication system is activated, which allows for videoconferencing between the patient in Morton and the physician in Peoria. The patient and physician hear and see one another in real time. The physician reviews the history and examination, discusses the assessment and plan with the patient, and provides patient education, making appropriate changes to the EMR as indicated based on the videoconference interaction.

\section{PATIENT SATISFACTION}

A prospective survey analysis determined patient satisfaction. A patient satisfaction survey developed by the US Department of Health and Human Services Health Resources and Services Administration was administered to all new patients upon check out from the INI Sleep Center-Morton (Sleep Center).

The survey included three specific questions that pertained to satisfaction with the "Provider":

- Listens to you

- Takes enough time with you

- Explains what you want to know

Each question was scored on a scale from 1 (lowest) to 5 (highest). The total patient satisfaction with provider score is the sum of the three questions regarding the provider, with a maximum score of 15 . Inclusion criteria for the study include subjects $\geq 18$ years of age at initial visit who were new patients at the Sleep Center and able to complete the survey. Exclusion criteria for the study include patients who were seen only by an NP and not the sleep specialist physician. Statistical analysis was performed using SAS 9.2 software.

\section{TREATMENT ADHERENCE}

Treatment adherence was investigated with a retrospective cohort study comparing the CPAP adherence of patients seen by the sleep physician via videoconference to patients seen by the sleep physician in person. The retrospective chart review identified adult subjects seen at the Sleep Center between November 2007 and September 2009 using the patient database available at the sleep center. 
Inclusion criteria for the study include subjects $\geq 18$ years of age at the initial visit subsequently diagnosed with OSA and prescribed CPAP. Exclusion criteria for the study include subjects who use CPAP brands other than ResMed (only one CPAP brand was selected for uniformity of reported adherence data) and those patients who were seen only by an NP and not the sleep specialist physician.

Subjects were divided into two groups: those who met the physician at their initial visit via videoconference and those who met the physician in person. Two variables were used to analyze CPAP adherence: percentage of nights CPAP was used for $\geq 4 \mathrm{~h}$ and the average number of minutes CPAP was used per night. The MannWhitney $t$-test was used to compare CPAP adherence between the groups over the most recent 14 consecutive days available. Statistical analysis was conducted using SAS 9.2 software.

\section{Results}

\section{PATIENT SATISFACTION}

Surveys were collected from 98 subjects from July 2009 to March 2010. Eight subjects were excluded: three did not complete the question regarding whether they met the physician in person or via video, two did not see a physician, two did not answer the questions regarding provider satisfaction, and one was $<18$ years of age.

Ultimately, 90 surveys were analyzed ( 56 subjects seen in person; 34 subjects seen via videoconference). The two groups were similar in gender, age, and ethnicity. Descriptive statistics are provided in Table 1.

\begin{tabular}{|c|c|c|}
\hline & IN PERSON & VIDEOCONFERENCE \\
\hline Number of patients $(n=90)$ & 56 & 34 \\
\hline \multicolumn{3}{|l|}{ Sex } \\
\hline Male $(\%)$ & 25 (44.64\%) & $14(42.42 \%)$ \\
\hline Female (\%) & $31(55.36 \%)$ & $19(57.58 \%)$ \\
\hline Not reported & & 1 \\
\hline \multicolumn{3}{|l|}{ Age } \\
\hline Mean (standard deviation) & $53.70(15.15)$ & $49.21(13.62)$ \\
\hline Median & 56 & 47.5 \\
\hline Minimum & 23 & 24 \\
\hline Maximum & 85 & 79 \\
\hline \multicolumn{3}{|l|}{ Ethnicity } \\
\hline White (\%) & $49(100 \%)$ & $24(92.30 \%)$ \\
\hline Asian (\%) & $0(0 \%)$ & $1(3.85 \%)$ \\
\hline Hispanic (\%) & $0(0 \%)$ & $1(3.85 \%)$ \\
\hline Not reported & 7 & 8 \\
\hline
\end{tabular}

\begin{tabular}{l|c|c}
\hline \multicolumn{3}{|c}{ Table 2. Mann-Whitney Test-Patient Satisfaction } \\
& IN PERSON $(\mathbf{N}=\mathbf{5 6})$ & $\begin{array}{c}\text { VIDEOCONFERENCE } \\
(\mathbf{N}=\mathbf{3 4})\end{array}$ \\
\begin{tabular}{l|c|} 
Mean patient satisfaction \\
score
\end{tabular} & 14.82 & 14.91 \\
\hline Standard deviation & 0.94 & 0.51 \\
\hline Minimum & 10 & 12 \\
\hline Maximum & 15 & 15 \\
\hline Mann-Whitney test & & \\
\hline$p$-Value & \multicolumn{2}{|c}{0.851} \\
\hline
\end{tabular}

The mean patient satisfaction scores of the two groups were analyzed using a Mann-Whitney test. The null hypothesis states that the means of the two groups are the same, whereas the alternative hypothesis states that the two groups are different. The null hypothesis was not rejected. Therefore, there is no difference between groups. Results are displayed in Table 2 and Figure 1.

\section{TREATMENT ADHERENCE}

Between November 2007 and September 2009, 987 new patients were seen at the Sleep Center (711 met the physician in person; 276 via videoconference). Of these, 174 subjects used a ResMed brand CPAP machine (111 in person; 63 via videoconference). Two subjects were excluded from the study: one was $<18$ years of age and one did not have adherence data in the database.

Data were analyzed for 172 subjects (111 in person; 61 via videoconference). The groups were similar in gender and age; descriptive statistics are provided in Table 3.

The Mann-Whitney test was used to analyze the two variables: percentage of nights CPAP was used $\geq 4 \mathrm{~h}$ and the average minutes of CPAP use per night. The null hypothesis for the test states that the means of the two groups are equivalent, whereas the alternative hypothesis states that the two groups are different. The null

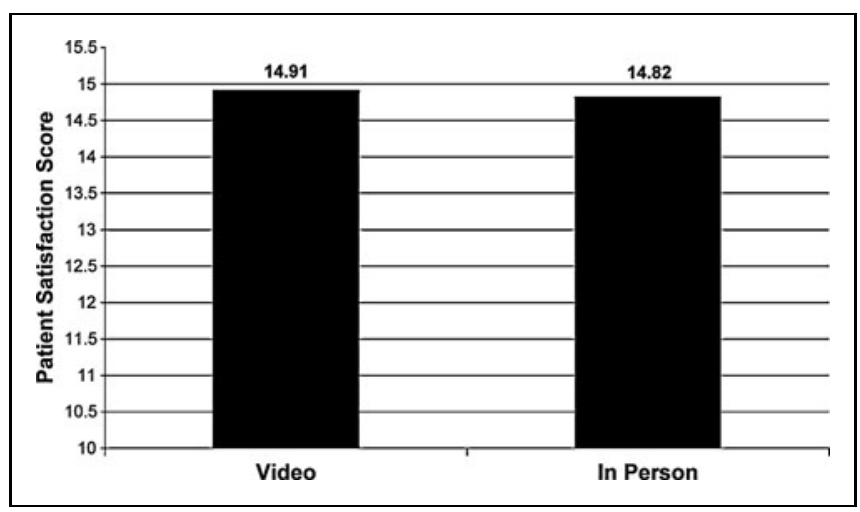

Fig. 1. Patient satisfaction score comparison. 


\begin{tabular}{l}
\multicolumn{3}{l}{ Table 3. Descriptive Statistics-Treatment Adherence } \\
\begin{tabular}{l|c|c} 
& IN PERSON & VIDEOCONFERENCE \\
\hline Subjects $(n=172)$ & 111 & 61 \\
\hline Sex & $68(61.26 \%)$ & $35(58.33 \%)$ \\
\hline Male (\%) & $43(38.74 \%)$ & $25(41.67 \%)$ \\
\hline Female (\%) & 0 & 1 \\
\hline Not reported & & \\
\hline Age & $55.66(13.36)$ & $56.26(15.74)$ \\
\hline Mean (standard deviation) & 57 & 56 \\
\hline Median & 23 & 18 \\
\hline Minimum & 91 & 92 \\
\hline Maximum &
\end{tabular}
\end{tabular}

hypothesis was not rejected in either analysis. Therefore, there is no difference between groups. Results from the statistical analysis are displayed in Tables 4 and 5 and Figures 2 and 3.

\section{Discussion}

Multiple studies have shown how various telemedicine techniques have been positively viewed by patients. Smith et al. provided at home CPAP education via telehealth services, which were positively viewed by most patients. ${ }^{6}$ Harrison et al. showed a positive patient perception of telecommunication tools in joint teleconsultation among the patient, the general practitioner, and a specialist. ${ }^{27}$

Patient adherence to CPAP for the management of OSA has been a longstanding struggle for sleep specialists. Studies have been done to determine rates of CPAP adherence and to establish predictors of adherence. Three different studies resulted in a varying range of $\mathrm{CPAP}$, with an average use ranging from $5.6 \mathrm{~h}$ per night to $6.2 \mathrm{~h}$ per night. Major predictors of CPAP compliance include subjective factors such as perceived daytime sleepiness (Epworth Score) and

\begin{tabular}{|c|c|c|}
\hline & $\begin{array}{l}\text { IN PERSON } \\
(N=111)\end{array}$ & $\begin{array}{l}\text { VIDEOCONFERENCE } \\
\qquad(\boldsymbol{N}=61)\end{array}$ \\
\hline Percentage of nights with use $\geq 4 \mathrm{~h}$ & 0.71 & 0.65 \\
\hline Standard deviation & 0.33 & 0.33 \\
\hline Minimum & 0.00 & 0.00 \\
\hline Maximum & 1.00 & 1.00 \\
\hline \multicolumn{3}{|l|}{ Mann-Whitney test } \\
\hline$p$-Value & & 0.198 \\
\hline
\end{tabular}

\begin{tabular}{|c|c|c|}
\hline & $\begin{array}{l}\text { IN PERSON } \\
(N=111)\end{array}$ & $\begin{array}{l}\text { VIDEOCONFERENCE } \\
\qquad(N=61)\end{array}$ \\
\hline Average minutes use per day & 340.55 & 305.31 \\
\hline Standard deviation & 149.77 & 154.07 \\
\hline Minimum & 3 & 0 \\
\hline Maximum & 655 & 579 \\
\hline \multicolumn{3}{|l|}{ Mann-Whitney test } \\
\hline$p$-Value & & 0.153 \\
\hline
\end{tabular}

snoring history and objective factors such as the apnea/hypopnea index and the oxygen desaturation index..$^{20,28,29}$ Smith et al. reported that providing patient education through telehealth services at home improves CPAP adherence (90\% after telehealth CPAP education vs. $44 \%$ without telehealth CPAP education) and that having the ability to monitor patients at home via telehealth services can prevent issues that would predispose people to nonadherence with their CPAP. ${ }^{6}$

To the best of our knowledge, the use of telehealth services at the initial consultation appointment in sleep medicine has not been previously studied with respect to either patient satisfaction or treatment adherence. This study showed that patient satisfaction and treatment adherence were no different between subjects who met the sleep physician in person and those who met the sleep physician via videoconferencing. This suggests that videoconferencing may be an acceptable and effective tool to increase access to care for patients with undiagnosed OSA.

The setting from which the physician and patient each originate may influence the perception or acceptance of telemedicine. Croteau et al. showed that physicians in urban areas tend not to be willing to dedicate time to learn how to use new telemedicine equipment;

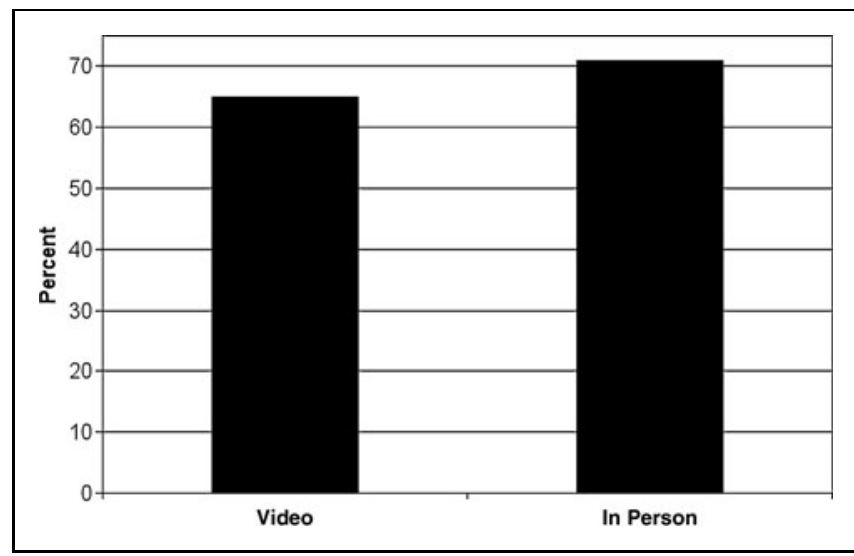

Fig. 2. Percentage of nights continuous positive airway pressure (CPAP) was used $\geq 4 \mathrm{~h}$. 


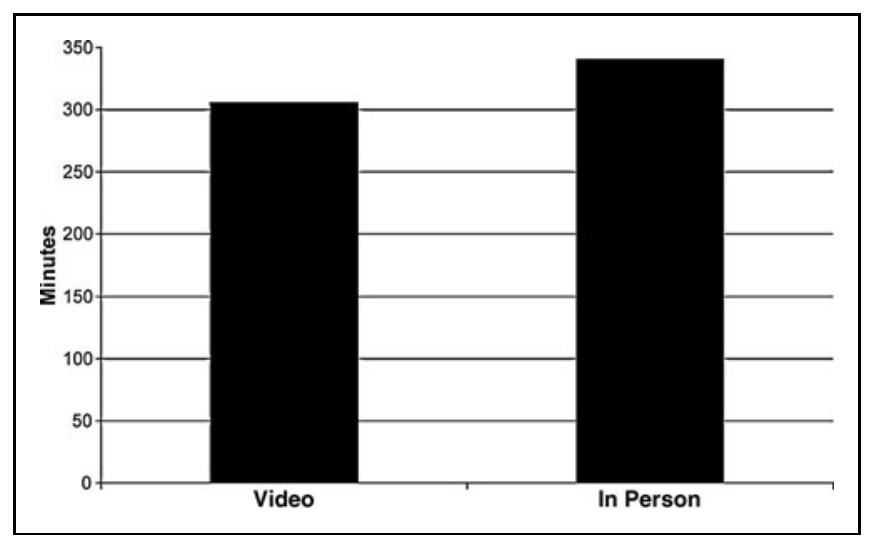

Fig. 3. Average minutes of CPAP use per night.

however, if the technology is easy to use the correlation between ease and implementation is positive. ${ }^{30}$ In a study of rural communities, Campbell et al. showed that physicians are more likely to adopt telemedicine technology if they perceive an increased capability of telemedicine to accommodate the constant advances of technology. In both rural and urban areas, perceived usefulness had the most significant impact on the decision to adopt telemedicine by healthcare providers. As long as telemedicine can be proven to be a useful tool for healthcare, the willingness of physicians to utilize telemedicine technology remains positive. ${ }^{31}$

In sleep medicine, the use of videoconferencing may be particularly helpful in two different settings. First, geographical areas where sleep specialist physicians are not available would benefit from videoconferencing tools to increase access to patient care. Rural communities, for example, may lack specialist services and may benefit from videoconferencing capabilities. Second, videoconferencing may assist clinical sleep medicine practices with multiple locations and a limited number of sleep specialist physicians. For example, this system has worked well at the sleep center with locations in Peoria and Morton, IL.

\section{LIMITATIONS OF THE STUDY}

In this study, all patients were initially seen by an NP (in person) followed by the sleep specialist physician (either in person or via videoconference). Whether CPAP adherence or patient satisfaction would differ with sleep specialist physician involvement versus the NP alone was not studied. This limitation was not tested because of the limited number of patients who are seen only by an NP at the initial visit.

This study had no means to compare patient preference of inperson versus televideo visits. The only comparison that could be made was between subjects. Each subject only had a single initial visit opportunity, and they were randomly chosen to see the physician either in person or via video.

This study also does not address the cost effectiveness of the patient meeting with the NP and sleep physician in person versus the sleep physician via videoconference. The costs of videoconferencing are not examined or compared with the costs of an in-person visit with the NP and sleep physician. Notably, telemedicine evaluation and management of patients is variably reimbursed by third-party payers, partly determined by the geographic location of the patient.

Videoconferencing differs from an in-person interaction both objectively and subjectively, and patient and physician impressions may differ. For example, the lack of physical contact (e.g., a handshake) may subtly change the perception of the personal nature of the visit. Either the patient or physician may perceive less time is available to communicate, even if the time is the same as in an in-person visit. The single-camera perspective of each person in the conversation limits the movement and angle of view of each participant. Sound quality via videoconferencing is likely to significantly differ than in an in-person conversation. These potential influences on the perception of teleconferencing were not specifically investigated in this study and may be worthy of further investigation.

This study indicates that subjects were equally satisfied with their provider interaction and adherent to CPAP treatment whether they saw the sleep physician in person or via videoconference. Therefore, videoconferencing may be an acceptable and effective tool to improve access to patient care.

Further study comparing patient satisfaction and CPAP adherence among those who saw only an NP with those who also saw a sleep physician may provide valuable additional information. Future study may also include cost analysis of seeing patients via videoconference versus in person, both in settings in which reimbursement for the visit is approved by third-party payers and those in which it is not. Finally, additional investigation into the numerous objective and subjective factors that can change both the patient and physicians' perception of a visit may be warranted.

\section{Acknowledgments}

The authors thank Aaron Taylor, Peggy Fisher, and Catherine Lipovsky for their assistance with this project.

\section{Disclosure Statement}

No competing financial interests exist.

\section{REFERENCES}

1. Dixon RF, Stahl JE. Virtual visits in a general medicine practice: A pilot study. Telemed J E Health 2008;14:525-530.

2. Morgan GJ, Craig B, Grant B, Sands A, Doherty N, Casey F. Home videoconferencing for patients with severe congenital heart disease following discharge. Congenit Heart Dis 2008;3:317-324.

3. Marrone $\mathrm{S}$, Mitchell JE, Crosby R, Wonderlich S, Jollie-Trottier T. Predictors of response to cognitive behavioral treatment for bulimia nervosa delivered via telemedicine versus face-to-face. Int J Eat Disord 2008;42:222-227.

4. King VL, Stollerv KB, Kidorf M, Kindbom K, Kursh S, Brady T, Brooner RK. Assessing the effectiveness of an Internet-based videoconferencing platform for delivering intensified substance abuse counseling. J Subst Abus Treat 2008;36:331-338.

5. Salvador $\mathrm{CH}$, Ruiz-Sanchez A, González de Mingo MA, Carmona Rodríguez M, Carrasco MP, Sagredo PG, Fragua JA, Caballero-Martinez F, García-López F, Márquez-Montes JN, Monteagudo JL. Evaluation of a telemedicine-based 


\section{PARIKH ET AL.}

service for the follow-up and monitoring of patients treated with oral anticoagulant therapy. IEEE Trans Inf Technol Biomed 2008;12:696-706.

6. Smith CE, Dauz ER, Clements F, Puno FN, Cook D, Doolittle G, Leeds W. Telehealth services to improve nonadherence: A placebo-controlled study. Telemed J E Health 2006;12:289-296.

7. Breslow MJ, Rosenfeld BA, Doefler M, Burke G, Yates G, Stone DJ, Tomaszewicz $P$, Hochman R, Plocher DW: Effect of a multiple-site intensive care unit telemedicine program on clinical and economic outcomes: An alternative paradigm for intensivist staffing. Crit Care Med 2004;32:31-38.

8. Waite $K$, Silver $F$, Jaigobin $C$, Black $S$, Lee L, Murray B, Danyliuk $P$, Brown EM. Telestroke: A multi-site, emergency-based telemedicine service in Ontario. J Telemed Telecare 2006;12:141-145.

9. Wallace $P$, Haines A, Harrison R, Barber J, Thompson S, Jacklin P, Roberts J, Lewis L, Wainwright $P$, Virtual Outreach Project Group. Joint Teleconsultations (virtual outreach) versus standard outpatient appointments for patients referred by their general practitioner for a specialist opinion: A randomised trial. The Lancet 2002;359:1961-1968.

10. Jacklin PB, Roberts JA, Wallace $P$, Haines $A$, Harrison $R$, Barber JA, Thompson SG, Lewis L, Currell R, Parker S, Wainwright P. Virtual outreach: economic evaluation of joint teleconsultations for patients referred by their general practitioner for a specialist opinion. BMJ 2003;327:84.

11. American Academy Sleep Medicine Force Task. Sleep-related breathing disorders in adults: recommendations for syndrome definition and measurement techniques in clinical research. Sleep 1999;22:667-689.

12. Aldrich MS. Sleep Medicine. Oxford: Oxford University Press, 1999.

13. Young $T$, Mari $P$, Jerome $D$, James $S$, Steven $W$, Safwan $B$. The occurrence of sleep-disordered breathing among middle-aged adults. $N$ Eng J Med 1993;328:1230-1235.

14. Shahar E, Whitney CW, Redline S, Lee ET, Newman AB, Javier Nieto F, O'Connor GT, Boland LL, Schwartz JE, Samet JM. Sleep-disordered breathing and cardiovascular disease: cross-sectional results of the sleep heart health study. Am J Respir Crit Care Med 2001;163:19-25.

15. Chervin RD, Murman DL, Malow BA, Totten V. Cost-utility of three approaches to the diagnosis of sleep apnea: Polysomnography, home testing, and empirical therapy. Ann Intern Med 1999;130:496-505.

16. Collop NA, Anderson WM, Boehlecke B, Claman D, Goldberg R, Gottlieb DJ, Hudgel D, Sateia M, Schwab R. Clinical Guidelines for the use of unattended portable monitors in the diagnosis of obstructive sleep apnea in adult patients. JSCM 2007;3:737-747.

17. Pépin $J$, Leger $P$, Veale $D$, Langevin $B$, Robert $D$, Lévy $P$. Side effects of nasal continuous positive airway pressure in sleep apnea syndrome: Study of 193 patients in two French Sleep Centers. Chest 1995;107:375-381.

18. Walker-Engström ML, Tegelberg A, Wilhelmsson B, Ringqvist I. 4-Year follow-up of treatment with dental appliance or uvulopalatopharyngoplasty in patients with obstructive sleep apnea: A randomized study. Chest 2003;121:739-746.

19. Peppard PE, Young T, Palta M, Dempsey J, Skatrud J. Longitudinal study of moderate weight change and sleep-disordered breathing. JAMA 2000;284:3015-3021.
20. McArdle N, Devereux G, Heidarnejad H, Engleman HM, Mackay TW, Douglas NJ. Long-term use of CPAP therapy for sleep apnea/hypopnea syndrome. Am J Respir Crit Care Med 1999;159:1108-1114.

21. Kristo DA, Andrada T, Eliasson AH, Poropatich RK, Netzer CM, Bradley JP, Loube DI, Netzer NC. Telemedicine in the Sleep Laboratory: Feasibility and economic advantages of polysomnograms transferred online. Telemed J E Health $2001 ; 7: 219-225$.

22. Kayyali HA, Weimer $S$, Frederick $C$, Martin $C$, Basa D, Juguilon JA, Jugilioni $F$. Remotely attended home monitoring of sleep disorders. Telemed $J$ E Health $2008 ; 14: 371-374$.

23. Buck $S$. Nine human factors contributing to the user acceptance of telemedicine applications: a cognitive-emotional approach. Telemed J E Health 2009;15:55-58.

24. Swinton JJ, Robinson WD, Bischoff RJ. Telehealth and rural depression: Physician and patient perspectives. Families, Systems, \& Health 2009;27:172-182.

25. Timmerberg RD, Wurst J, Patterson J, Spaulding RJ, Belz NE. Feasibility of using videoconferencing to provide diabetes education: a pilot study. Telemed J $E$ Health 2009;15:95-97.

26. Williams JBW, Ellis A, Middleton A, Kobak KA. Primary care patients in psychiatric clinical trials: a pilot study using videoconferencing. Ann Gen Psychiatry 2007;6:24.

27. Harrison R, MacFarlane A, Murray E, Wallace P. Patients' perceptions of joint teleconsultations: A qualitative evaluation. Health Expect 2005;9:81-90.

28. Krieger J, Kurtz D, Petiau C, Sforza E, Trautman D. Long-term compliance with CPAP therapy in obstructive sleep apnea patients and in snorers. Sleep 1996;19(9 Suppl):S136-S143.

29. Kohler M, Smith D, Tippett V, Strandlings JR. Predictors of long-term compliance with continuous positive airway pressure. Thorax 2010;65: 829-832.

30. Croteau AM, Vieru D. Telemedicine adoption by different groups of physicians. Proceedings of the 35th Hawaii International Conference on System Sciences, (HICSS '02). 2002;6:151-159.

31. Campbell JD, Harris KD, Hodge R. Introducing telemedicine technology to rural physicians and settings. J Fam Pract 2001;50:419-424.

Address correspondence to: Roshni Parikh, M.D. University of Illiniois College of Medicine-Neurology 1 Illini Drive Peoria, IL 61602

E-mail: roshni.parikh06@gmail.com

Received: January 30, 2011 Accepted: March 14, 2011 